\title{
miR-145-5p is associated with pathological complete response to neoadjuvant chemotherapy and impairs cell proliferation by targeting TGF $\beta R 2$ in breast cancer
}

\author{
FREDERIK GARCÍA-GARCÍA ${ }^{1}$, YARELY M. SALINAS-VERA ${ }^{1}$, RAÚL GARCÍA-VÁZQUEZ ${ }^{2}$, \\ LAURENCE A. MARCHAT ${ }^{2}$, SERGIO RODRÍGUEZ-CUEVAS ${ }^{3}$, JOSÉ SULLIVAN LÓPEZ-GONZÁLEZ ${ }^{4}$, \\ ÁNGELES CARLOS-REYES ${ }^{4}$, ROSALÍO RAMOS-PAYÁN ${ }^{5}$, MARIBEL AGUILAR-MEDINA ${ }^{5}$, \\ CARLOS PÉREZ-PLASENCIA ${ }^{6}$, ERIKA RUÍZ-GARCÍA ${ }^{7}$ and CÉSAR LÓPEZ-CAMARILLO ${ }^{1}$ \\ ${ }^{1}$ Genomics Sciences Program, Autonomous University of Mexico City, Mexico City 03100; \\ ${ }^{2}$ Molecular Biomedicine Program and Biotechnology Network, Instituto Politécnico Nacional, Mexico City 07320; \\ ${ }^{3}$ Breast Diseases Institute, FUCAM, Mexico City 04980; ${ }^{4}$ Lung Cancer Laboratory, National Institute of Respiratory \\ Diseases 'Ismael Cosio Villegas', Mexico City $14080 ;{ }^{5}$ Faculty of Sciences Chemistry Biologicals, \\ Autonomous University of Sinaloa, Culiacán Sinaloa 80040; ${ }^{6}$ Genomics Laboratory, National Institute of Cancerology; \\ ${ }^{7}$ Translacional Medicine Laboratory, National Institute of Cancer, Mexico City 14080, Mexico
}

Received May 30, 2018; Accepted March 19, 2019

DOI: $10.3892 / o r .2019 .7102$

\begin{abstract}
Cancer patients who better benefit from neoadjuvant chemotherapy (NeoCh) are those who achieve a successful pathological complete response ( $\mathrm{pCR}$ ) represented by the absence of residual disease. Unfortunately, no highly sensitive and specific tumor biomarkers for predicting the clinical response to $\mathrm{NeoCh}$ have yet been defined. The aim of the present study was to ascertain whether miR-145-5p could discriminate between pCR and no-pCR in triple-negative breast cancer patients that received a cisplatin/doxorubicin-based neoadjuvant treatment. miR-145-5p expression was determined in breast tumors by quantitative RT-PCR. Our data showed that miR-145-5p had a significant low expression $(\mathrm{P}<0.005)$ in patients that achieved pCR in comparison to the non-responder group. Kaplan Meier analysis indicated that low levels of miR-145-5p were associated with increased disease-free survival. In addition, receiver operating characteristic (ROC) curve analysis suggested that miR-145-5p is a good predictor of pCR $(\mathrm{P}<0.003, \mathrm{AUC}=0.7899,95 \% \mathrm{CI}, 0.6382-0.9416)$. Quantitative RT-PCR expression analysis also revealed that miR-145-5p was downregulated in four breast cancer cell lines relative to normal cells. To study the functions of miR-145-5p, its expression was restored in triple-negative MDA-MB-231
\end{abstract}

Correspondence to: Dr César López-Camarillo, Genomics Sciences Program, Autonomous University of Mexico City, San Lorenzo 290, Col. Del Valle, Mexico City 03100, Mexico E-mail: genomicas@yahoo.com.mx

Key words: breast cancer, pathological complete response, neoadjuvant therapy, miR-145-5p, cell proliferation, apoptosis, TGF $\beta$ R2 cells and its effects in cell proliferation were evaluated by MTT assays and in apoptosis using Annexin V experiments. Data revealed that ectopic expression of miR-145-5p resulted in a significant inhibition of cell proliferation and also induced apoptosis. Moreover, miR-145-5p led to sensitization of breast cancer cells to cisplatin therapy. In addition, western blot assays indicated that miR-145-5p downregulated the TGF $3 \mathrm{R} 2$ protein. In conclusion, miR-145-5p could be a potential biomarker of clinical response to $\mathrm{NeoCh}$ in triple-negative breast cancer. Functionally miR-145-5p may regulate cell proliferation, at least in part, by targeting TGF $\beta R 2$.

\section{Introduction}

Pathological complete response (pCR) is defined by the rate of the absence of residual invasive breast cancer disease after preoperative neoadjuvant chemotherapy ( $\mathrm{NeoCh}$ ), and pCR has been used as the primary end point in many neoadjuvant trials (1). NeoCh constitutes an important standard approach for locally advanced breast cancer, and it takes place before surgical extraction of tumors with the objective of reducing high tumor size (2). This procedure aims to render locally advanced cancers operable, to facilitate the removal of tumors, to allow breast-conserving surgery, and to improve postoperative recovery and long-term outcome for the patients $(3,4)$. In addition, neoadjuvant trials provide the opportunity to test new drugs preoperatively in patients with locally invasive breast cancer accordingly to subtypes and hormonal receptor status (5-7). Large multi-centric studies have stablished that patients who better benefit from NeoCh are those who achieve a successful pCR which has been associated with both improved disease-free survival (DFS) and overall survival (OS) rates (8). For example, the CTNeoBC study in a large cohort of women with breast cancer identified a good association between pCR 
and DFS/OS (9). However, no specific and sensitive biomarkers to predict the clinical response to $\mathrm{NeoCh}$ in breast cancer have yet been defined.

MicroRNAs (miRNAs) are evolutionarily conserved single-stranded tiny non-coding RNAs of 21-25 nucleotides in length that function as negative post-transcriptional regulators of gene expression (10). The mechanism of action of miRNAs relies in the partially complementary binding with the $3^{\prime}$ untranslated region (UTR) of specific target mRNAs resulting in either translation inhibition or deadenylation-dependent degradation of encoding protein transcripts in the cytoplasmic P-bodies. Thus, these small RNAs function as guide molecules in post-transcriptional gene silencing. miRNAs have normal functions in eukaryotic cells including cell growth, differentiation, survival and metabolism. However, alterations in the transcriptional and epigenetic mechanisms leading to aberrant expression of miRNAs and its target genes have been frequently observed in breast cancer. To date, there is sufficient experimental evidence which strongly links miRNAs with the development and progression of breast cancer, as they function as oncomiRs through the regulation of tumor-suppressor genes and cellular oncogenes (11). Moreover, changes in the abundance of miRNAs have been associated with clinical and pathological features of patients. Notably, miRNAs have also been recently investigated as potential predictors of clinical response to cytotoxic therapy in diverse types of cancers (12-19). Previously, we reported a miRNA expression signature associated with pathological complete response to NeoCh in triple negative breast cancer patients (19). In the present study, we focused on the clinical and molecular analysis of miR-145-5p, as its relationships with response to therapy have not been addressed in triple negative breast cancer. Our data strongly suggest that miR-145-5p could be a predictor of $\mathrm{pCR}$ to NeoCh in breast cancer patients. Moreover, the present experimental findings demonstrated a potential function for miR-145-5p as a regulator of cell proliferation and apoptosis in breast cancer cells.

\section{Materials and methods}

Statement of ethics. The Breast Cancer Foundation (FUCAM) of Mexico provided the breast tumors and normal tissue collection. The Ethics Committee of the Breast Cancer Foundation (FUCAM) of Mexico approved the protocols using human tissues. Signed informed consent forms were obtained from the participants prior to release for research use. This study was carried out in accordance with the ethical standards of the committee and in accordance with the Helsinki Declaration of 1975.

Tissue samples. Formalin-fixed paraffin-embedded (FFPE) tissues from triple-negative breast cancer patients $(n=32)$ who received neoadjuvant cisplatin/doxorubicin-based chemotherapy at FUCAM between November 2008 and August 2017 were collected. Tumor samples were classified as with or without pathological complete response (pCR) to neoadjuvant therapy. Patients were aged between 28 and 65 years; mean age was 46 years in the pCR group and 53 in the no-pCR group. Pathologist confirmed the existence of at least $80 \%$ tumor cells in the clinical specimens.
Cell lines. Human breast cancer cell lines were obtained from the American Type Culture Collection (ATCC; Manassas, VA, USA). MCF-7 (ATCC: HTB-22), MDA-MB-231 (ATCC: HTB-26), SKBR3 (ATCC: HTB-30), BT-20 (ATCC: HTB19) and no-tumorigenic MCF-10A (ATCC: CRL-10317) were routinely grown in Dulbecco's modified Eagle's minimal essential medium (DMEM; Gibco; Thermo Fisher Scientific, Inc., Waltham, MA, USA), supplemented with $10 \%$ fetal bovine serum (FBS) and penicillin-streptomycin $(50 \mathrm{U} / \mathrm{ml}$; Invitrogen; Thermo Fisher Scientific, Inc.) in $5 \% \mathrm{CO}_{2}$ atmosphere at $37^{\circ} \mathrm{C}$.

Reverse transcription and real-time polymerase chain reaction. Five serial $20-\mu$ m-thick sections of FFPE tissue specimens were used for total RNA isolation using the RNeasy FFPE kit (Qiagen Inc., Valencia, CA, USA) with modifications to the manufacturer's protocol. Briefly, the sections were incubated twice in xylene for $1 \mathrm{~h}$ at $63^{\circ} \mathrm{C}$ for deparaffinization followed by purification of total RNA using TRIzol protocol (Ambion; Thermo Fisher Scientific, Inc.). RNA concentration and purity were analyzed for spectrophotometry (NanoDrop Technologies; Thermo Fisher Scientific, Inc.), and integrity was evaluated by $1 \%$ agarose gel electrophoresis. Quantitative RT-PCR (qRT-PCR) assay of individual miR-145 was performed using MicroRNA assays (cat. no. 4427975; Thermo Fisher Scientific, Inc.). Briefly, 10 ng total RNA were reverse transcribed using a stem looped-RT specific primer, $0.15 \mu \mathrm{l}$ dNTPs $(100 \mathrm{mM})$, $1.0 \mu 1$ reverse transcriptase MultiScribe (Thermo Fisher Scientific, Inc.) (50 U/ $\mu 1), 1.5 \mu 1$ 10X buffer, $0.19 \mu 1$ RNase inhibitor $(20 \mathrm{U} / \mu \mathrm{l})$ and $4.16 \mu \mathrm{l}$ RNase-free water. Then, retrotranscription reaction (1:15 dilution) was mixed with $10 \mu 1$ TaqMan Universal PCR Master Mix, No AmpErase UNG 2X, $7.67 \mu 1$ RNase-free water and 1.0 $\mu$ l PCR probe. PCR reaction was performed in a GeneAmp System 9700 (Applied Biosystems; Thermo Fisher Scientific, Inc.) as follows: $95^{\circ} \mathrm{C}$ for $10 \mathrm{~min}$, and 40 cycles at $95^{\circ} \mathrm{C}$ for $15 \mathrm{sec}$ and $60^{\circ} \mathrm{C}$ for $1 \mathrm{~min}$. Tests were normalized using RNU44 as internal control. Experiments were performed three times in triplicate, and the results were expressed as mean $\pm \mathrm{SD}$. Relative quantification was referred as $\Delta \Delta \mathrm{Cq}$ as previously described (20). $\mathrm{P}<0.05$ was considered to indicate statistical significance.

miR-145-5p restoration in breast cancer cells. miR-145-5p mimics $(60 \mathrm{nM}$, GUCCAGUUUUCCCAGGAAUCCCU; Thermo Fisher Scientific, Inc.) and scramble sequence used as a negative control (60 nM, AM17110; Thermo Fisher Scientific, Inc.) were individually transfected into MDA-MB-231 cells using siPORT amine transfection agent (Ambion; Thermo Fisher Scientific, Inc.). Briefly, miR-145-5p and scramble were added to wells containing $1 \times 10^{7}$ cells and incubated for $48 \mathrm{~h}$. Then, total RNA was extracted using TRIzol and miR-145-5p restoration was evaluated by qRT-PCR using specific stem-looped RT oligonucleotide and TaqMan probe (4427975; Thermo Fisher Scientific, Inc.) as implemented in the TaqMan MicroRNA Assay protocol. Experiments were performed three times in triplicate and the results are expressed as mean $\pm \mathrm{SD}$. $\mathrm{P}<0.05$ was considered to indicate statistical significance. 
Cell proliferation assays. Cell proliferation was measured using the 3-(4,5-dimethylthiazol-2-yl)-2,5-diphenyltetrazolium bromide (MTT) assay (5 $\mathrm{mg} / \mathrm{ml}$; Sigma-Aldrich; Merck KGaA, Darmstadt, Germany). MTT reagent was added to the MDA-MB-231 cells ( $1 \times 10^{5} /$ well) transfected with mimic miR-145-5p or scramble and mock and incubated for $3.5 \mathrm{~h}$ at $37^{\circ} \mathrm{C}$. Then, dissolution buffer (99\% isopropanol) was added to the cells and incubated for an additional $15 \mathrm{~min}$. Absorbance was recorded at $12 \mathrm{~h}$ using a spectrophotometer $(570-630 \mathrm{~nm})$. Data were analyzed using BioStat software (AnalystSoft, Inc. Walnut, CA, USA). Experiments were performed three times in triplicate and the results are expressed as mean \pm SD. $\mathrm{P}<0.05$ was considered to indicate statistical significance.

Fluorescence-activated cell sorting assays (FACS). MDA-MB-231 cells $\left(2 \times 10^{5}\right)$ were treated for $48 \mathrm{~h}$ with siPORT transfection agent (mock), scramble $(60 \mathrm{nM})$ and mimic miR-145-5p (60 nM). Cisplatin $\left(56 \mu \mathrm{M}, \mathrm{IC}_{50}\right)$ was added to the miR-145-5p mimic-transfected cells and to the non-transfected cells and incubated by $24 \mathrm{~h}$. Subsequently, cells were harvested, washed twice with phosphate-buffered saline (PBS) $1 \mathrm{X}$ and resuspended in $100 \mu \mathrm{l}$ buffer $(10 \mathrm{mMHEPES}, 140 \mathrm{mM} \mathrm{NaCl}$ and $2.5 \mathrm{mM} \mathrm{CaCl}_{2}$ ), and processed for apoptosis assays and FACS following the manufacturer's instructions (Annexin V-FLUOS staining kit; Roche Diagnostics, Basel, Switzerland). Briefly, the cells were stained with $2 \mu \mathrm{l}$ Annexin V-FITC and $2 \mu \mathrm{l}$ propidium iodide (PI) mixed with $100 \mu 1$ incubation buffer for $15 \mathrm{~min}$, washed with $500 \mu \mathrm{l}$ binding buffer and resuspended in $300 \mu \mathrm{l}$ PBS 1X. Apoptosis events were evaluated using the FACSCalibur flow cytometer [BD Immunocytometry Systems (BDIS); BD Biosciences, Franklin Lakes, NJ, USA). Briefly, Annexin V and PI emissions were detected in the FL-1 and FL-2 channels, respectively. For each sample, data from 20,000 cells were acquired in list mode on logarithmic scales. Data were analyzed using the Summit V4.3 software (Dako; Agilent Technologies, Inc., Santa Clara, CA, USA) and the results are represented as the total percentage of apoptotic cells as the sum of both early and late phases of apoptosis (Annexin V-FITC-positive). Assays were performed in triplicate and data are expressed as mean $\pm \mathrm{SD}$. $\mathrm{P}<0.05$ was considered to indicate statistical significance.

Bioinformatic prediction of miR-145-5p gene targets. miR-145 target genes were predicted using TargetScan v.7.2 (http://www. targetscan.org/vert_72/), miRWalk v.2.0 (http://mirwalk.umm. uni-heidelberg.de/) and PicTar (https://pictar.mdc-berlin.de/) software. Only those gene targets predicted by the three algorithms were included in downstream analysis. Cellular pathways and processes potentially affected by miR-145-5p were predicted using DAVID v.6.7 software (https://david.ncifcrf.gov/).

Kaplan-Meier analysis. Kaplan-Meier method was used to evaluate the disease-free survival (DFS) associated with miR-145-5p expression in breast cancer patients. The significance of the survival differences was determined by the log-rank test with $95 \%$ confidence intervals (CI).

Western blot analysis. Whole protein extracts from MDA-MB-231 cells transfected with miR-145-5p (60 nM) mimic, scramble $(60 \mathrm{nM})$ or mock were obtained using
TNTE buffer (50 mM TRIS-HCl pH 7.4, 150 mM NaCl, 0.5\% Triton X-100 and $5 \mathrm{mM}$ EDTA) supplemented with complete protease inhibitor cocktail (Roche Molecular Biochemicals, Penzberg, Upper Bavaria, Germany). Protein extracts (40 $\mu \mathrm{g}$ ) were separated by $10 \%$ SDS-PAGE and electrotransferred to nitrocellulose membrane (Bio-Rad Laboratories, Hercules, CA, USA). After blocking with 5\% non-fat dry milk and $0.05 \%$ Tween-20 in PBS pH 7.4 overnight at $4^{\circ} \mathrm{C}$, the membranes were probed with the TGF $\beta R 2$ antibody (dilution 1:500; cat. no. ab78419; Abcam, Cambridge, UK) overnight at $4^{\circ} \mathrm{C}$. For detection, the membranes were incubated with peroxidase-conjugated goat anti-mouse secondary antibodies (dilution 1:2,000; cat. no. G-21040; Molecular Probes; Thermo Fisher Scientific, Inc.) in 5\% non-fat dry milk and $0.05 \%$ Tween-20 in PBS pH 7.4 and immunocomplexes were developed using the ECL chemiluminescence system (Amersham Pharmacia Biotech, Little Chalfont, UK). Membranes were subjected to striping and re-blotting with GAPDH monoclonal antibodies (dilution 1:2,000; cat. no. sc-47724; Santa Cruz Biotechnology, Santa Cruz, CA, USA). Densitometric analysis of immunodetected bands in western blots assays was performed using the public domain ImageJ software (https://imagej.nih.gov/ij/index.html).

Statistical analysis. A t-test was used to identify significant differences in miRNA expression between patients with pathological complete response to chemotherapy treatment in comparison to the non-responder group. For parametric data we used one-way analysis of variance (ANOVA) to compare between groups. A $\mathrm{P}<0.05$ was considered as statistically significant. GraphPad Prism v.5 was used for statistical anlaysis (https://www.graphpad.com/scientific-software/prism/). Experiments were performed three times in triplicate and the results were represented as mean \pm standard deviation (SD).

\section{Results}

Low miR-145-5p levels are associated with response to chemotherapy and higher disease-free survival. Breast cancer tumors were collected from a cohort of patients $(n=32)$ diagnosed with locally advanced triple-negative breast cancer. Tissues were tested by immunohistochemistry to confirm the triple-negative status. To evaluate whether changes in miR-145-5p expression levels could identify the breast cancer patients that achieved pCR from no-responder individuals, we set up stem-loop reverse transcription-quantitative PCR (RT-PCR) experiments. Our data from the $2^{-\Delta \Delta \mathrm{Cq}}$ analyses showed that miR-145-5p was differentially expressed between both groups (Fig. 1A). miR-145-5p exhibited a significantly low expression $(\mathrm{P}<0.0047)$ in patients that achieved a pathological complete response (pCR) to chemotherapy treatment in comparison to the non-responder group. Kaplan-Meier survival analysis for miR-145-5p expression was performed to estimate the disease-free survival (DFS) in the triple-negative breast cancer patients. Patients were dichotomized at their median into two groups with low and high miR-145-5p expression according to quantile expression. The log-rank (Mantel-Cox) test identified significant differences $(\mathrm{P}<0.0007)$ between the groups of patients with a median survival of 104 months in the pCR group (95\% CI, 0.03462-0.2513). Breast cancer patients 

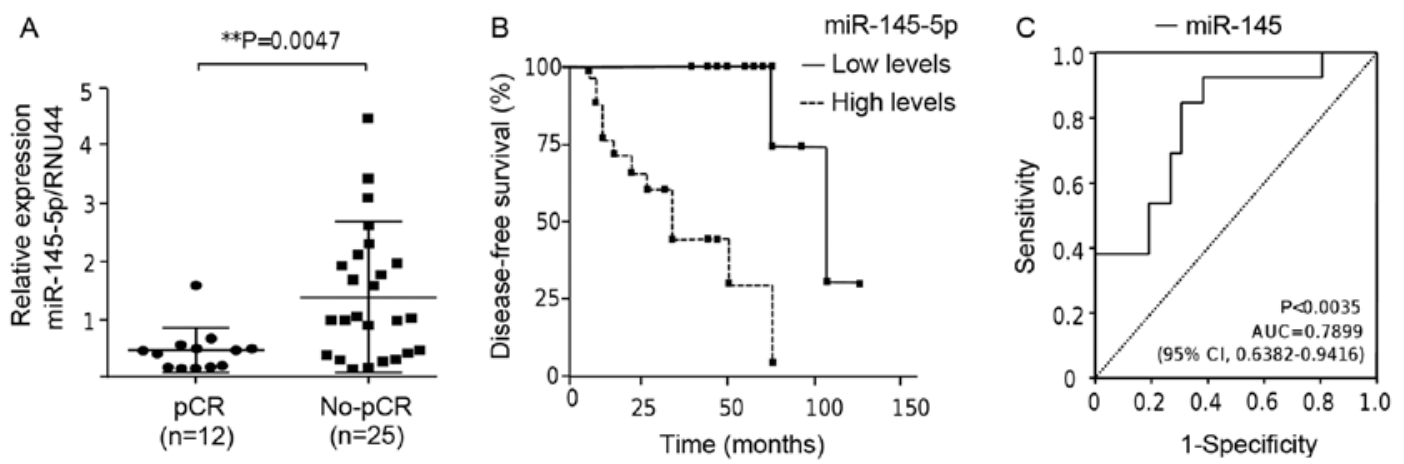

Figure 1. (A) Relative expression of miR-145-5p in patients with ( $\mathrm{n}=12$ ) pathological complete response (pCR) or without $\mathrm{pCR}$ (n=25) (no-pCR) to neoadjuvant chemotherapy. Data were normalized with RNU44 values. Data were analyzed using the Student's t-test. ${ }^{* * *} \mathrm{P}=0.0047$. (B) Kaplan-Meier analysis of disease-free survival for patients with low and high levels of miR-145-5p. (C) ROC curve of miR-145-5p expression. ROC, receiver operating characteristic; AUC, area under curve; CI, confidence interval.
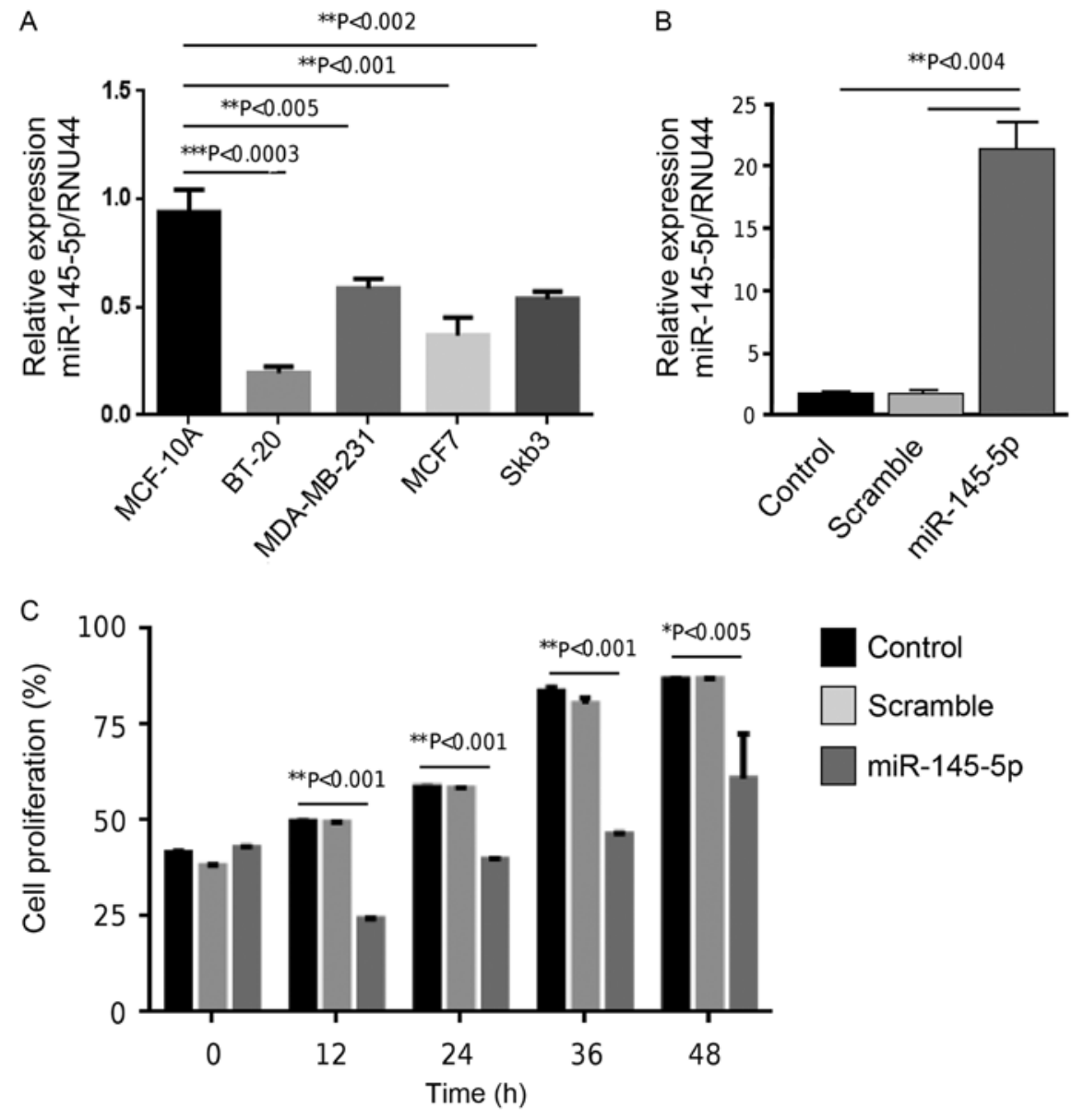

Control

Scramble

$\operatorname{miR}-145-5 p$

Figure 2. (A) miR-145-5p expression in breast cancer cell lines and non-tumorigenic cells. (B) Validation of miR-145-5p mimics in MDA-MB-231 cells. (C) MTT assays in cells transfected with miR-145-5p, scramble and without transfection. Data were analyzed using one-way analysis of variance (ANOVA) to compare between groups.

with low levels of miR-145-5p had a higher DFS in comparison to the patients with high levels of miR-145-5p (Fig. 1B). In contrast, breast cancer patients with high levels of miR-145-5p did not respond to the chemotherapy regimen and had a worst outcome. Moreover, receiver operating characteristic (ROC) curve analysis suggested that miR-145-5p could be a predictor of pCR. The area under the curve (AUC) was 0.7899 ( $\mathrm{P}<0.0035$, 95\% CI, 0.6382-0.9416) (Fig. 1C).
miR-145-5p is downregulated in breast cancer cell lines and impairs cell proliferation. To investigate the biological relevance of miR-145-5p, its expression was evaluated in breast cancer cells using stem-loop RT-PCR assays. Data showed that miR-145-5p expression was significantly downregulated in BT-20, MDA-MB-231, MCF-7 and SK-BR-3 breast cancer cell lines in comparison to MCF-10A normal mammary cells (Fig. 2A). We next aimed to ascertain whether 
A
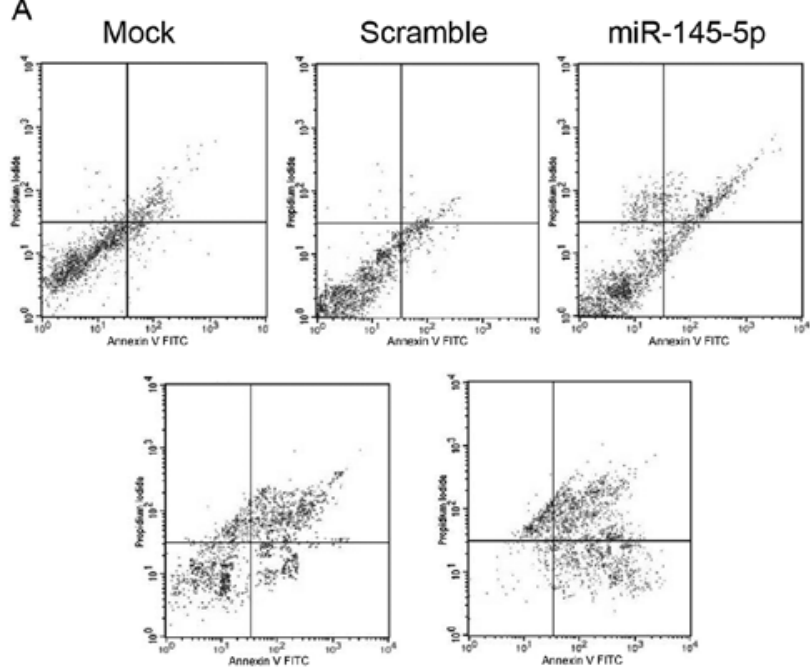

Cisplatin

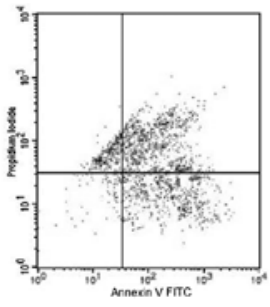

miR-145-5p

plus cisplatin
B

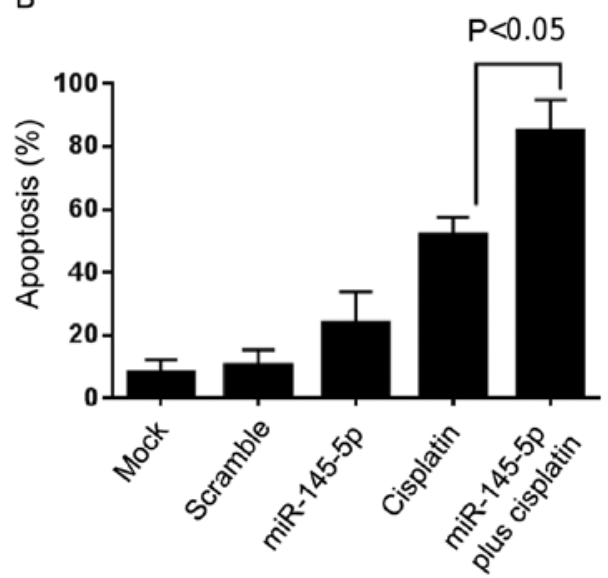

Figure 3. miR-145-5p induces apoptosis. (A) Annexin V assays of miR-154-5p-transfected cells and controls. (B) Graphical representation of assays in A. Data were analyzed using one-way ANOVA to compare between groups. Bars represents the mean of three independent experiments $\pm \mathrm{SD}$. $\mathrm{P}<0.05$.

forced expression of miR-145-5p has negative effects on cell proliferation using MTT assays. BT-20 cells were initially used for miR-145-5p analysis. However, after RNA mimic transfection, we repeatedly observed a large decrease in cell viability $(80 \%)$ indicating an effect on this cell line which impeded to continue with further characterization (data not shown). Thus, we decided to use the MDA-MB-231 cells as a model for functional assays as also it exhibited a significant and important downregulation of miR-145-5p expression. Transfection of miR-145-5p mimics (30 nM) was effective to restore the expression by 20 -fold in MDA-MB-231 cells in comparison to the non-transfected and scramble negative control transfected cells (Fig. 2B). Cell proliferation assay data indicated that the growth rate of triple-negative MDA-MB-231 cells transfected with precursor miR-145-5p (60 nM) was significantly $(\mathrm{P}<0.001)$ decreased at an early $(12 \mathrm{~h})$ to late $(48 \mathrm{~h})$ time of incubation in comparison with the mock and scramble control cells (Fig. 2C).

miR-145-5p restoration induces apoptosis and sensitizes breast cancer cells to cisplatin therapy. It was next evaluated whether miR-145-5p overexpression results in apoptosis activation. For this purpose Annexin $\mathrm{V}$ assays and fluorescence-activated cell sorting were performed. Results showed that treatment with miR-145-5p mimics was able to induce a modest but significant increase in apoptosis of MDA-MB-231 cells relative to the mock and scramble-transfected controls (Fig. 3A). Then, we evaluated the effect of miR-145-5p in the response to cisplatin in MDA-MB-231 breast cancer cells. Both precursor miR-145-5p-transfected and non-transfected cells were submitted to cisplatin $\left(\mathrm{IC}_{50} 55 \mu \mathrm{M}\right)$ monotherapy for 48 h. Notably, combined dual therapy using miR-145-5p plus cisplatin induced a synergistic increase $(\mathrm{P}<0.05)$ in the early and late apoptosis of MDA-MB-231 cells in comparison to cisplatin alone (Fig. 3B). These data indicate that miR-145-5p sensitizes breast cancer cells to cisplatin therapy.
miR-145-5p modulates diverse oncogenic signaling pathways. In order to obtain insight concerning the molecular mechanisms of miR-145-5p in breast cancer, a bioinformatic analysis of potential target genes was performed using TargetScan 7.2, miRWalk v.2.0 and PicTar softwares as described in Materials and methods. Our computational analysis identify 1,007 potential gene targets of miR-145-5p many of them involved in the regulation of diverse transducers with pivotal functions in oncogenic signaling pathways including TGF $\beta$, PI3K/AKT, ErbB, VEGF/MAPK, FOXO, FAK, JAK/STAT and mTOR (Fig. 4). Collectively these signaling transduction pathways may regulate apoptosis, cell cycle, migration, metastasis and survival of tumor cells which highlights the potential tumor-suppressor functions of miR-145-5p. Of these pathways, we focused on the study of TGF $\beta$ due to its role as an oncogenic pathway at the advanced stages of disease.

miR-145-5p downregulates TGF $\beta R 2$ protein. TGF $\beta$ signaling functions as a function of tumor suppressors or oncogenes during early and late stages of carcinogenesis, respectively (21). Our bioinformatic analyses identified two potential miR-145-5p binding sites in the 3'UTR of the TGF $\beta$ R2 gene (Fig. 5A). Given its importance in the positive regulation of cancer hallmarks, we decided to ascertain whether miR-145-5p exerts a potential posttranscriptional repression of TGF $\beta$ R2. For this purpose, western blot assays were performed using specific antibodies and whole protein extracts from MDA-MB-231 cells transfected with precursor miR-145-5p and non-transfected mock and scramble-transfected controls. Our data revealed that miR-145-5p mimics resulted in a significant $(\mathrm{P}<0.05)$ and severe downregulation of TGF $\beta \mathrm{R} 2$ protein levels in comparison to mock and scramble controls (Fig. 5B and C). GADPH levels used as control did not show significant changes after miR-145-5p treatment (Fig. 5B). These data indicate that miR-145-5p negatively regulates TGF $\beta R 2$ by a direct or indirect mechanism. 


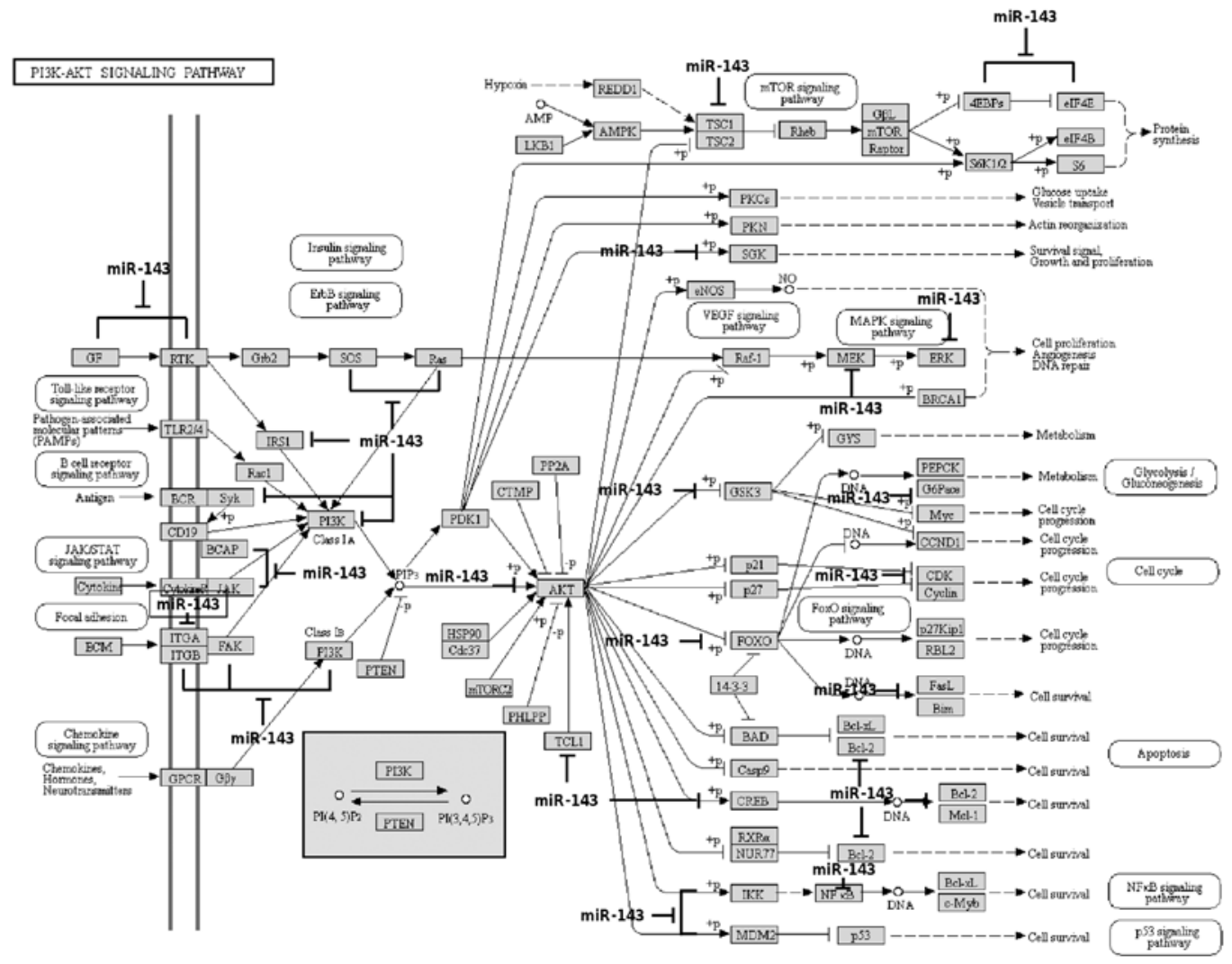

Figure 4. miRNA-mRNA interactions. Pathways in cancer modulated by deregulated miR-145-5p are depicted. KEGG tool algorithm was used to predict the interactions $(\mathrm{P}<0.05)$. KEGG, Kyoto Encyclopedia of Genes and Genomes.

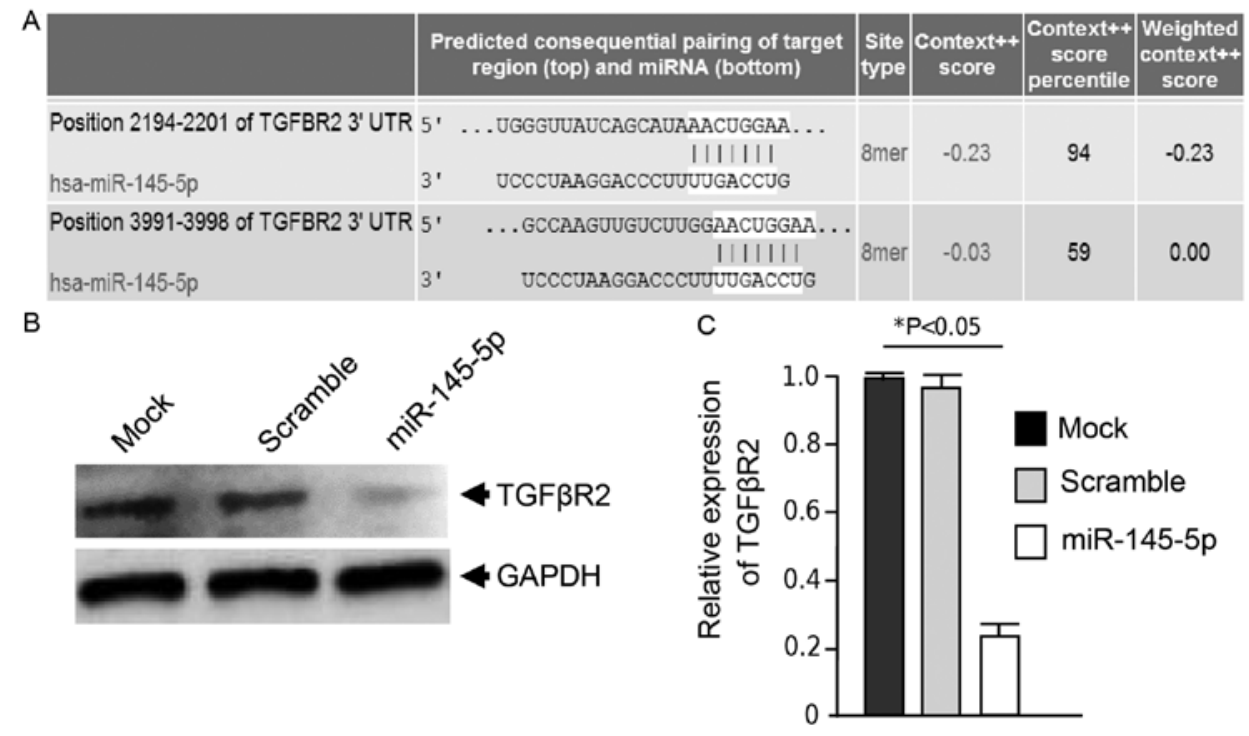

Figure 5. Western blot analysis of TGFßR2. (A) Predictions of miR-145-5p binding sites in 3'UTR of TGFßR2. (B) Western blot analysis of transfected cells with miR-145-5p and controls. (C) Densitometric analysis of bands in B. Data were analyzed using one-way ANOVA to compare between groups. Data are represented as the mean $\pm \mathrm{SD}$. UTR, $3^{\prime}$ untranslated region. ${ }^{*} \mathrm{P}<0.05$.

\section{Discussion}

Currently, there are no effective methods to identify breast cancer patients who would benefit from neoadjuvant therapy. Thus, there is a need to identify novel biomarkers that may predict clinical response to therapy and will achieve a pathological complete response. Recent studies revealed that changes in miRNA expression could be associated with successful pathological complete response (pCR) after neoadjuvant treatment in different types of human cancers (5-7). In order to contribute to the scarce list of potential predictors of clinical response to chemotherapy in breast cancer, in the present study, analysis of miR-145-5p was focused on as a potential predictor for $\mathrm{pCR}$ to $\mathrm{NeoCh}$ in triple-negative breast cancer patients. First, it was shown that low miR-145-5p levels were significantly associated with $\mathrm{pCR}$ to neoadjuvant 
therapy. Receiver operating characteristic (ROC) curve analysis suggested that miR-145-5p could be a predictor of pCR $(\mathrm{P}<0.0035, \mathrm{AUC}=0.7899 ; 95 \% \mathrm{CI}, 0.6382-0.9416)$. Moreover, low levels of miR-145-5p also predicted a higher disease-free survival (Fig. 1). These data are relevant and highlight the potential of miR-145-5p as a biomarker of response to therapy.

It is important to note that during chemotherapy treatments, miRNA dynamics is a complex event. Before $\mathrm{NeoCh}$, we distinguish two groups of patients: i) a group with very low miR-145 (low miR-145-5p); and ii) a group with relative high expression (high miR-145-5p). Remarkably, both groups of cancer patients had lower expression of miR-145-5p in comparison to normal cell lines (Fig. 2A) and tissues, as expected for a tumor-suppressor gene. In our cohort of patients, relative high expression of miR-145-5p before the therapy was associated with a worse response to chemotherapy (no-pCR) (Fig. 1A) and low disease-free survival (DFS) in comparison to the miR-145-5p low group (Fig. 1B). These data confirm our previous global miRNA profiling findings in pCR and no-pCR patients treated with a novel chemotherapeutic regimen (19). In addition, it has been reported that several miRNAs with known oncogenic or tumor-suppressor functions frequently exhibit significant up- and down-variations in their expression levels before, during and after chemotherapy, indicating the profound effect of drugs in miRNA regulation. Therefore, alterations in miRNA abundance are not always associated with its functions in carcinogenesis studied in vitro with cell lines, although the possibility of an unknown dual function cannot be ruled out (e.g. at early stages of tumorigenesis TGF $\beta$ is a tumor suppressor, but at the late stages of disease it acts as a potent oncogene). These observations may reflect the complexity of therapy resistance observed in vivo with cancer patients, which also have been pointed out by other authors investigating miRNAs as potential predictors of clinical response to neoadjuvant therapies. Thus, the fact that low levels of miR-145-5p were associated with a good response to neoadjuvant therapy may be inconsistent with the tumor-suppressive role reported for miR-145-5p. Since miR-145-5p targets many genes, its functions may differ by diverse biological and therapeutic events (e.g. in vivo vs. in vitro; adjuvant chemotherapy vs. chemo-radiotherapy; the percentage of patients achieving pCR vs. patients without response, neoadjuvant vs. no-neoadjuvant regimen). Indeed, consistent with our findings, recent studies have shown a potential role of miR-145 associated with resistance to chemo-radiotherapy. For instance, similar data were reported in locally advanced rectal cancer patients in whom low levels of miR-145 and miR-143 predicted pCR to neoadjuvant chemo-radiotherapy (22), indicating that miR-145 and miR-143 levels may be novel, non-invasive predictive markers of response to therapy in cancer patients. Thus, we propose that in vivo variations in miR-145 levels before and after therapy may not always reflect the functions observed in isolated cancer cell lines. Further analyses are needed to confirm the miR-145 levels in large cohorts of patients and its biological roles associated with resistance to therapy.

It was also found that miR-145-5p expression was significantly repressed in breast tumors and in four breast cancer cell lines relative to normal mammary tissues and non-tumorigenic cells, respectively (Fig. 2A). At the functional level, miR-145 regulates tumor growth, cell proliferation, apoptosis, cell migration and invasion in breast cancer cells (23-28). In agreement with its tumor-suppressor functions reported in vitro, here it was revealed that ectopic restoration of miR-145-5p inhibited cell proliferation in triple-negative MDA-MB-231 cells and sensitized tumor cells to cisplatin treatment reinforcing the notion that miR-145-5p is a bona fide tumor suppressor associated with therapy response (Fig. 2). Intriguingly, miR-145-5p RNA mimic transfection in BT-20 cells induced a large decrease in cell viability. We hypothesized that the BT-20 cell genetic background may influence and exacerbate the cell response to miR-495. Although this cell line is also triple negative it contains important sequence variations in master genes controlling cell proliferation and survival including PI3KCA, CDKN2A, EGFR and p53 (29), that may exert an unknown effect in response to miR-145-5p restoration; however, further investigation is needed to support these assumptions.

Novel data concerning the miR-145-5p mechanisms associated with cancer hallmark inhibition is provided as the findings elucidated that miR-145 directly or indirectly targets TGF $\beta$ signaling in breast cancer cells. During the preparation of this manuscript a recent study reported that miR-145 inhibits cell proliferation by targeting TGF $\beta 1$ in breast cancer cells (30). In the present study, we added a piece in the puzzle of miR-145-5p functions and demonstrated that it also targets TGF $\beta$ R 2 which strengthens the notion that this tiny non-coding RNA has a profound impact on the suppression of cancer hallmarks through modulation of TGF $\beta$ signaling in breast cancer. In summary, our data suggest that miR-145-5p could be a potential predictor of response to neoadjuvant therapy, and also elucidate the molecular functions of miR-145-5p in breast cancer cells.

\section{Acknowledgements}

We acknowledge Consejo Nacional de Ciencia y Tecnología CONACyT, México and Universidad Autónoma de la Ciudad de México for support.

\section{Funding}

The present study was funded by the Consejo Nacional de Ciencia y Tecnología CONACyT México, Fondo SSA/IMSS/ ISSSTE (grant no. 233370 and 222335).

\section{Availability of data and materials}

The datasets used during the present study are available from the corresponding author upon reasonable request.

\section{Authors' contributions}

CLC, LAM, SRC and ERG conceived and designed the study. SRC provided the tissues collection. FGG, YMSV, RGV and ACR performed the experiments. CLC, CPP, RRP, YMSV, RGV and MAM analyzed the data. CLC, FGG and LAM wrote the paper. ERG, RRP, MAM and JSLG reviewed and edited the manuscript. JSLG, ACR, CPP and SRC provided the additional materials and reagents. All authors read and approved the manuscript and agree to be accountable for all aspects of the 
research in ensuring that the accuracy or integrity of any part of the work are appropriately investigated and resolved.

\section{Ethics approval and consent to participate}

The Breast Cancer Foundation (FUCAM) of Mexico provided the breast tumors and normal tissue collection. The Ethics Committee of the Breast Cancer Foundation (FUCAM) of Mexico approved the protocols using human tissues. Signed informed consent forms were obtained from the participants prior to release for research use. This study was carried out in accordance with the ethical standards of the committee and in accordance with the Helsinki Declaration of 1975.

\section{Patient consent for publication}

Not applicable.

\section{Competing interests}

The authors declare that they have no competing interests.

\section{References}

1. Prowell TM and Pazdur R: Pathological complete response and accelerated drug approval in early breast cancer. $\mathrm{N}$ Engl J Med 366: 2438-2441, 2012

2. Von Minckwitz G, Untch M, Blohmer JU, Costa SD, Eidtmann H, Fasching PA, Gerber B, Eiermann W, Hilfrich J, Huober J, et al: Definition and impact of pathologic complete response on prognosis after neoadjuvant chemotherapy in various intrinsic breast cancer subtypes. J Clin Oncol 30: 1796-1804, 2012.

3. Liedtke C, Mazouni C, Hess KR, André F, Tordai A Mejia JA, Symmans WF, Gonzalez-Angulo AM, Hennessy B, Green M, et al: Response to neoadjuvant therapy and long-term survival in patients with triple negative breast cancer. J Clin Oncol 26: 1275-1281, 2008.

4. Kong X, Moran MS,Zhang N,Haffty B and Yang Q: Meta-analysis confirms achieving pathological complete response after neoadjuvant chemotherapy predicts favourable prognosis for breast cancer patients. Eur J Cancer 47: 2084-2090, 2011.

5. Tanioka M, Sasaki M, Shimomura A, Fujishima M, Doi M, Matsuura K, Sakuma T, Yoshimura K, Saeki T, Ohara M, et al: Pathologic complete response after neoadjuvant chemotherapy in HER2-overexpressing breast cancer according to hormonal receptor status. Breast 23: 466-472, 2014

6. Zhang P, Yin Y, Mo H, Zhang B, Wang X, Li Q, Yuan P, Wang J, Zheng S, Cai R, et al: Better pathologic complete response and relapse-free survival after carboplatin plus paclitaxel compared with epirubicin plus paclitaxel as neoadjuvant chemotherapy for locally advanced triple-negative breast cancer: A randomized phase 2 trial. Oncotarget 7: 60647-60656, 2016.

7. Luangdilok S, Samarnthai N and Korphaisarn K: Association between pathological complete response and outcome following neoadjuvant chemotherapy in locally advanced breast cancer patients. J Breast Cancer 17: 376-385, 2014.

8. Ring AE, Smith IE, Ashley S, Fulford LG and Lakhani SR: Oestrogen receptor status, pathological complete response and prognosis in patients receiving neoadjuvant chemotherapy for early breast cancer. Br J Cancer 91: 2012-2017, 2004.

9. Cortazar P,Zhang L, Untch M, Mehta K, Costantino JP, Wolmark N, Bonnefoi H, Cameron D, Gianni L, Valagussa P, et al: Pathological complete response and long-term clinical benefit in breast cancer: The CTNeoBC pooled analysis. Lancet 384: 164-172, 2014.

10. Rupaimoole R and Slack FJ: MicroRNA therapeutics: Towards a new era for the management of cancer and other diseases. Nat Rev Drug Discov 16: 203-222, 2017.

11. Esquela-Kerscher A and Slack FJ: Oncomirs-microRNAs with a role in cancer. Nat Rev Cancer 6: 259-269, 2006.

12. Kolacinska A, Morawiec J, Fendler W, Malachowska B Morawiec Z, Szemraj J, Pawlowska Z, Chowdhury D, Choi YE, Kubiak R, et al: Association of microRNAs and pathologic response to preoperative chemotherapy in triple negative breast cancer: Preliminary report. Mol Biol Rep 41: 2851-2857, 2014.
13. Hummel R, Hussej DJ and Haier J: MicroRNAs: Predictors and modifiers of chemo- and radiotherapy in different tumor types. Eur J Cancer 46: 298-311, 2010.

14. Gasparini P, Cascione L, Fassan M, Lovat F, Guler G, Balci S, Irkkan C, Morrison C, Croce CM, Shapiro CL and Huebner K: microRNA expression profiling identifies a four microRNA signature as a novel diagnostic and prognostic biomarker in triple negative breast cancers. Oncotarget 5: 1174-1184, 2014.

15. Ohzawa H, Miki A, Teratani T, Shiba S, Sakuma Y, Nishimura W, Noda Y, Fukushima N, Fujii H, Hozumi Y, et al: Usefulness of miRNA profiles for predicting pathological responses to neoadjuvant chemotherapy in patients with human epidermal growth factor receptor 2-positive breast cancer. Oncol Lett 13: 1731-1740, 2017.

16. Raychaudhuri M, Bronger H, Buchner T, Kiechle M, Weichert W and Avril S: MicroRNAs miR-7 and miR-340 predict response to neoadjuvant chemotherapy in breast cancer. Breast Cancer Res Treat 162: 511-521, 2017.

17. Pedroza-Torres A, Fernández-Retana J, Peralta-Zaragoza O, Jacobo-Herrera N, Cantú de Leon D, Cerna-Cortés JF, Lopez-Camarillo C and Pérez-Plasencia C: A microRNA expression signature for clinical response in locally advanced cervical cancer. Gynecol Oncol 142: 557-565, 2016.

18. Petrillo M, Zannoni GF, Beltrame L, Martinelli E, DiFeo A, Paracchini L, Craparotta I, Mannarino L, Vizzielli G, Scambia G, et al: Identification of high-grade serous ovarian cancer miRNA species associated with survival and drug response in patients receiving neoadjuvant chemotherapy: A retrospective longitudinal analysis using matched tumor biopsies. Ann Oncol 27: 625-634, 2016.

19. García-Vazquez R, Ruiz-García E, Meneses García A, Astudillo-de la Vega H, Lara-Medina F, Alvarado-Miranda A, Maldonado-Martínez H, González-Barrios JA, Campos-Parra AD, Rodríguez Cuevas $\mathrm{S}$, et al: A microRNA signature associated with pathological complete response to novel neoadjuvant therapy regimen in triple-negative breast cancer. Tumour Biol 39: 1010428317702899, 2017.

20. Livak KJ and Schmittgen TD: Analysis of relative gene expression data using real-time quantitative PCR and the $2^{-\Delta \Delta C T}$ method. Methods 25: 402-408, 2001.

21. Syed V: TGF- $\beta$ signaling in cancer. J Cell Biochem 117: 1279-1287, 2016.

22. Hiyoshi Y, Akiyoshi T, Inoue R, Murofushi K, Yamamoto N, Fukunaga Y, Ueno M, Baba H, Mori S and Yamaguchi T: Serum miR-143 levels predict the pathological response to neoadjuvant chemoradiotherapy in patients with locally advanced rectal cancer. Oncotarget 8: 79201-79211, 2017.

23. Zhao H, Kang X, Xia X, Wo L, Gu X, Hu Y, Xie X, Chang H, Lou L and Shen X: miR-145 suppresses breast cancer cell migration by targeting FSCN-1 and inhibiting epithelial-mesenchymal transition. Am J Transl Res 8: 3106-3114, 2016.

24. Gao M, Miao L, Liu M, Li C, Yu C, Yan H, Yin Y, Wang Y, Qi X and Ren J: miR-145 sensitizes breast cancer to doxorubicin by targeting multidrug resistance-associated protein-1. Oncotarget 7: 59714-59726, 2016.

25. Zheng M, Wu Z, Wu A, Huang Z, He N and Xie X: MiR-145 promotes TNF- $\alpha$-induced apoptosis by facilitating the formation of RIP1-FADD caspase-8 complex in triple-negative breast cancer. Tumour Biol 37: 8599-8607, 2016.

26. Yan X, Chen X, Liang H, Deng T, Chen W, Zhang S, Liu M, Gao X, Liu Y, Zhao C, et al: miR-143 and miR-145 synergistically regulate ERBB3 to suppress cell proliferation and invasion in breast cancer. Mol Cancer 13: 220, 2014.

27. Götte M, Mohr C, Koo CY, Stock C, Vaske AK, Viola M, Ibrahim SA, Peddibhotla S, Teng YH, Low JY, et al: miR-145-dependent targeting of junctional adhesion molecule A and modulation of fascin expression are associated with reduced breast cancer cell motility and invasiveness. Oncogene 29: 6569-6580, 2010.

28. Wang S, Bian C, Yang Z, Bo Y, Li J, Zeng L, Zhou H and Zhao RC: miR-145 inhibits breast cancer cell growth through RTKN. Int J Oncol 34: 1461-1466, 2009.

29. Saunus JM, Smart CE, Kutasovic JR, Johnston RL, Kalita-de Croft P, Miranda M, Rozali EN, Vargas AC, Reid LE, Lorsy E, et al: Multidimensional phenotyping of breast cancer cell lines to guide preclinical research. Breast Cancer Res Treat 167: 289-301, 2018.

30. Ding Y, Zhang C, Zhang J, Zhang N, Li T, Fang J, Zhang Y, Zuo F, Tao Z, Tang S, et al: miR-145 inhibits proliferation and migration of breast cancer cells by directly or indirectly regulating TGF- $\beta 1$ expression. Int J Oncol 50: 1701-1710, 2017. 